\title{
Optimal design of fractional order linear system with stochastic inputs/parametric uncertainties by hybrid spectral method
}

\author{
Pham Luu Trung Duong, Moonyong Lee* \\ School of Chemical Engineering, Yeungnam University, Gyeongsan, Republic of Korea
}

\section{A R T I C L E I N F O}

\section{Article history:}

Received 20 November 2013

Received in revised form 14 June 2014

Accepted 6 August 2014

Available online 10 September 2014

\section{Keywords:}

Block pulse function

Fractional calculus

Operational matrix

Stochastic collocation

Polynomial chaos

\begin{abstract}
A B S T R A C T
This paper reports the design of a fractional linear system under stochastic inputs/uncertainties. The design methods were based on the hybrid spectral method for expanding the system signals over orthogonal functions. The use of the hybrid spectral method led to algebraic relationships between the first and second order stochastic moments of the input and output of a system. The spectral method could obtain a highly accurate solution with less computational demand than the traditional Monte Carlo method. Based on the hybrid spectral framework, the optimal design was elaborated by minimizing the suitably defined constrained-optimization problem.
\end{abstract}

(c) 2014 Elsevier Ltd. All rights reserved.

\section{Introduction}

In recent years, fractional calculus has attracted considerable attention and is becoming increasingly popular because of its practical applications in a range of science and engineering fields [1-5]. This is because mathematical models based on fractional derivatives can describe a variety of natural phenomena, such as flexible structures [6], anomalous system [7], and viscoelastic materials [8]. On the other hand, fractional order systems are often studied using models with fixed deterministic parameters and inputs. Moreover, the input/parameters of these models are uncertain due to the inherent variability and/or incomplete knowledge. Therefore, the development of methods capable of designing fractional order systems with uncertainties is necessary.

The well-known Monte Carlo (MC) method is a typical approach for simulating stochastic models $[9,10]$. This method involves the generation of independent realizations of random inputs based on their prescribed probability distribution. For each realization, the data is fixed and the problem becomes deterministic. Solving the multiple deterministic realizations builds an ensemble of solutions, i.e. the realization of random solutions, from which

\footnotetext{
* Corresponding author at: School of Chemical Engineering, Yeungnam University, Gyeongsan 712-749, Republic of Korea. Tel.: +82 53810 3241; fax: +82 538113262. E-mail address: mynlee@yu.ac.kr (M. Lee).
}

statistical information can be extracted, e.g. mean and variance. This approach is simple to apply, involving only repeated deterministic simulations, but the convergence is slow and large numbers of calculations are required. For example, the mean values typically converge as $1 / \sqrt{M}$, where $M$ is the number of realizations.

Generalized polynomial chaos (gPC) [11-13] is a more recent approach for quantifying the uncertainty within system models. On the other hand, to simulate stochastic systems using the gPC method, the random inputs of many systems involve random processes approximated by truncated Karhunen-Loeve (KL) expansion, and the dimensionality of the input depends on the correlation lengths of these processes. For an input with a low correlation length (ideal white noise), the number of dimensions required for an accurate representation can be large, which increases the computational demand substantially using the gPC method.

A recent study [14] introduced a hybrid spectral method for quantifying the uncertainties in single input single output (SISO) fractional order systems. This paper extends the framework in Ref. [14] for the optimal design of a fractional SISO system under stochastic input/parametric uncertainty.

This paper is organized as follows: Section 2 briefly introduces a hybrid spectral method for uncertainty quantification in fractional order systems. Section 3 defines the suitable performance objectives coupled with the spectral method for the design of a stochastic linear fractional system. Section 4 considers examples ranging from integer to fractional order to demonstrate the proposed method. 


\section{Fractional order system}

This section summarizes the main concepts, definitions and basic results from fractional calculus, which are useful for further developments.

\subsection{Governing equation for system dynamics}

Among the many formulations of the generalized derivative with non-integer order, the Riemann-Liouville definition is used most commonly [15]

$D_{0}^{\alpha} f(t)=\frac{1}{\Gamma(m-\alpha)}\left(\frac{d}{d t}\right)^{m} \int_{0}^{t} \frac{f(\tau)}{(t-\tau)^{1-(m-\alpha)}} d \tau$,

where $\Gamma(x)$ denotes the gamma function; $m$ is the integer satisfying $m-1<\alpha<m$.

The Riemann-Liouville fractional integral of a function $f(t)$ is defined as

$I_{0}^{\alpha} f(t)=\frac{1}{\Gamma(\alpha)} \int_{0}^{t} \frac{f(\tau)}{(t-\tau)^{1-\alpha}} d \tau$

The Laplace transform for a fractional order derivative under zero initial conditions is defined as

$L\left\{D_{0}^{\alpha} f(t)\right\}=s^{\alpha} F(s)$,

where $F(s)$ is the Laplace transform of $f(t)$.

Therefore, a fractional order single input single output (SISO) system can be described by the following fractional order differential equation

$$
\begin{aligned}
a_{0} D_{0}^{\alpha_{0}} y(t)+ & a_{1} D_{0}^{\alpha_{1}} y(t)+\cdots+a_{l} D_{0}^{\alpha_{l}} y(t)=b_{0} D_{0}^{\beta_{0}} u(t) \\
& +b_{1} D_{0}^{\beta_{1}} u(t)+\cdots+b_{m} D_{0}^{\beta_{m}} u(t),
\end{aligned}
$$

or by the transfer function,

$G(s)=\frac{Y(s)}{U(s)}=\frac{b_{m} s^{\beta_{m}}+\cdots+b_{0} s^{\beta_{0}}}{a_{l} s^{\alpha_{l}}+\cdots+a_{0} s^{\alpha_{0}}}$

where $\alpha_{i}$ and $\beta_{i}$ are the arbitrary real positive numbers, and $u(t)$ and $y(t)$ are the system's input and output, respectively.

\subsection{Operational matrices of block pulse function for analysis of fractional order system}

Block pulse functions are a complete set of orthogonal functions that are defined over the time interval, $[0, \tau]$,

$\psi_{i}=\left\{\begin{array}{ll}1 & \frac{i-1}{N} \tau \leq t \leq \frac{i}{N} \tau \\ 0 & \text { elsewhere }\end{array}\right.$.

where $N$ is the number of block pulse functions.

Therefore, any function that can be absolutely integrated on the time interval $[0, \tau]$ can be expanded into a series from the block pulse basis:

$f(t)=\psi_{N}^{T}(t) C_{f}=\sum_{i=1}^{N} c_{f_{i}} \psi_{i}(t)$,

where $\boldsymbol{\psi}_{N}^{T}(t)=\left[\psi_{1}(t), \ldots, \psi_{N}(t)\right]$ constitutes of the block pulse basis. From here, the subscript, $N$, of $\boldsymbol{\psi}_{N}^{T}(t)$ is dropped out for the convenience of notation.
The expansion coefficients (or spectral characteristics) can be calculated as follows

$c_{f_{i}}=\frac{N}{\tau} \int_{[(i-1) / N] \tau}^{(i / N) \tau} f(t) \psi_{i}(t) d t$.

Furthermore, any function $g\left(t_{1}, t_{2}\right)$ absolutely integrable over the time interval, $[0, \tau] \times[0, \tau]$, can be expanded as

$g\left(t_{1}, t_{2}\right)=\sum_{i=1}^{N} \sum_{j=1}^{N} c_{i j} \psi_{i}\left(t_{1}\right) \psi_{j}\left(t_{2}\right)=\boldsymbol{\psi}^{T}\left(t_{1}\right) C_{g} \psi\left(t_{2}\right)$.

with expansion coefficients (or spectral characteristics) of

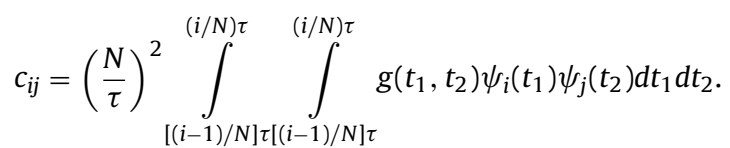

Eq. (2) can be expressed in terms of the operational matrix [16]

$I_{0}^{\alpha} f(t)=\psi(t)^{T} A_{\alpha} C_{f}$.

where the generalized operational matrix integration of the block pulse function, $A_{\alpha}$, is

$A_{\alpha}=P_{\alpha}^{T}=\left(\frac{\tau}{N}\right)^{\alpha} \frac{1}{\Gamma(\alpha+2)}\left(\begin{array}{ccccc}f_{1} & f_{2} & f_{3} & \ldots & f_{N} \\ 0 & f_{1} & f_{2} & \ldots & f_{N-1} \\ \vdots & \ddots & \ddots & \ddots & \vdots \\ 0 & \ldots & \ldots & \ldots & f_{1}\end{array}\right)^{T}$.

The elements of the generalized operational matrix integration can be expressed as

$f_{1}=1 ; f_{p}=p^{\alpha+1}-2(p-1)^{\alpha+1}+(p-2)^{\alpha+1}$ for $p=2,3 \ldots$

The generalized operational matrix of a derivative of order $\alpha$ is

$B_{\alpha} A_{\alpha}=I$,

where $I$ is the identity matrix.

The generalized operational matrix of the derivative can be used to approximate Eq. (1) as follows:

$D_{0}^{\alpha} f(t)=\psi(t)^{T} B_{\alpha} C_{f}$

Using the operational matrix of the fractional order derivative, Eq. (4) can be rewritten in the following form:

$A_{G}=\left(a_{l} D_{\alpha_{l}}+\cdots+a_{0} D_{\alpha_{0}}\right)^{-1}\left(b_{m} D_{\beta_{m}}+\cdots+b_{0} D_{\beta_{0}}\right)$.

The input and output of the system is thus linked by

$C_{Y}=A_{G} C_{U} ; \quad Y(t)=\left(C_{Y}\right)^{T} \boldsymbol{\psi}(t) ; \quad U(t)=\left(C_{U}\right)^{T} \boldsymbol{\psi}(t)$.

Closed-loop control systems normally comprise several elements, such as the controller and plant in Figs. 1 and 2, in terms of the transfer function and operational matrix, respectively. The operational matrix of a closed-loop system can be found using block diagram algebra similar to the block algebra used for the transfer function [10].

More on detail on the operational matrix with respect to the different polynomial functions can be found in $[16,17]$ and the references therein. 


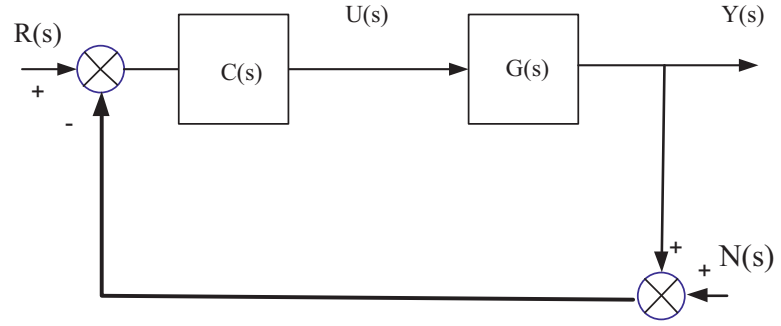

Fig. 1. Closed-loop control system.

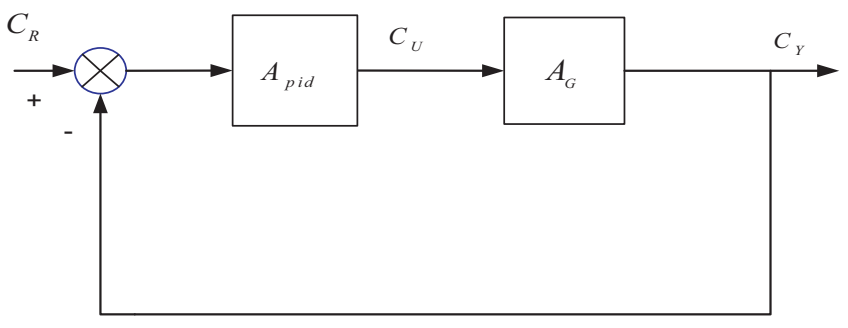

Fig. 2. Closed-loop control system in terms of operational matrices.

\subsection{Stochastic analysis of fractional order systems}

Consider the system described by Eq. (4) with random independent coefficients, $a_{0}, \ldots, a_{l}, b_{0} \ldots, b_{m}$, with the given distributions. The spectral characteristics of its input and output are linked by Eq. (17). Assume that the random forcing input with a given mean and covariance function is as follows:

$M_{U}(t)=E[U(t)]=\left(C_{m_{U}}\right)^{T} \psi(t)$

$\kappa_{U U}=E\left\{\left[U\left(t_{1}\right)-M_{U}\left(t_{1}\right)\right]\left[U\left(t_{2}\right)-M_{U}\left(t_{2}\right)\right]\right\}=\sum_{i=1}^{N} \sum_{j=1}^{N} \psi_{i}\left(t_{1}\right) \psi_{j}\left(t_{2}\right) c_{i j}=\boldsymbol{\psi}\left(t_{1}\right)^{T} C_{K_{U U}} \psi\left(t_{2}\right)$.

After some manipulation of the operational matrix, the spectral characteristics of the mean and covariance of the system's output can be expressed as [14]

$C_{m_{Y}}=E\left[A_{G}\right] C_{m_{U}}$

$C_{\kappa_{Y Y}}=E\left[A_{G}\left\{C_{\kappa_{U U}}+\left(C_{m_{U}}\right)\left(C_{m_{U}}\right)^{T}\right\} A_{G}\right]^{T}-C_{m_{Y}}\left(C_{m_{Y}}\right)^{T}$

or,

$m_{Y}(t)=\boldsymbol{\psi}(t)^{T} E\left[A_{G}\right] C_{m_{U}}$

$\kappa_{Y Y}\left(t_{1}, t_{2}\right)=\boldsymbol{\psi}\left(t_{1}\right)^{T} E\left[A_{G}\left\{C_{\kappa_{U U}}+\left(C_{m_{U}}\right)\left(C_{m_{U}}\right)^{T}\right\} A_{G}^{T} \boldsymbol{\psi}\left(t_{2}\right)\right]-\boldsymbol{\psi}\left(t_{1}\right)^{T} C_{m_{Y}}\left(C_{m_{Y}}\right)^{T} \boldsymbol{\psi}\left(t_{2}\right)$

Random parameters, $a_{i}, b_{j}$, result in the random operational matrix, $A_{G}$, in Eqs. (19) and (20), and its moment can be estimated using a stochastic collocation [14].

When parameters $a_{i}, b_{j}$ are deterministic, Eq. (20) becomes

$m_{Y}(t)=\boldsymbol{\psi}(t)^{T} A_{G} C_{m_{U}}$

$\kappa_{Y Y}\left(t_{1}, t_{2}\right)=\boldsymbol{\psi}\left(t_{1}\right)^{T} A_{G}\left\{C_{\kappa_{U U}}+\left(C_{m_{U}}\right)\left(C_{m_{U}}\right)^{T}\right\} A_{G}^{T} \boldsymbol{\psi}\left(t_{2}\right)-\boldsymbol{\psi}\left(t_{1}\right)^{T} C_{m_{Y}}\left(C_{m_{Y}}\right)^{T} \boldsymbol{\psi}\left(t_{2}\right)$

\section{Optimal design of fractional order system with stochastic input/parametric uncertainties}

(a) Shaping filter design by parametric optimization
A shaping filter is normally used to shape the covariance function of a given input noise into to a desired noise with a reference covariance.

Assume that the system is described by Eq. (4), where the coefficients $a_{i}, b_{j}, \alpha_{k}, \beta_{l}$ are the deterministic design parameters. The input is a random process with a zero mean and given covariance function. The mean and covariance function of the input can be expanded to a series of BFF, as expressed in Eq. (18). Therefore, the mean and covariance function for the output are given by Eq. (21). The covariance of desired is given and expanded in spectral form as follows:

$$
\begin{aligned}
\kappa_{Y_{D} Y_{D}}\left(t_{1}, t_{2}\right)= & E\left\{\left[Y_{D}\left(t_{1}\right)-M_{Y_{D}}\left(t_{1}\right)\right]\left[Y_{D}\left(t_{2}\right)-M_{Y_{D}}\left(t_{2}\right)\right]\right\} \\
& =\sum_{i=1}^{N} \sum_{j=1}^{N} \psi_{i}\left(t_{1}\right) \psi_{j}\left(t_{2}\right) c_{i j}^{\kappa} Y_{D}^{Y_{D}}=\psi\left(t_{1}\right)^{T} C_{K_{Y_{D} Y_{D}}} \psi\left(t_{2}\right) .
\end{aligned}
$$

From Eq. (21) it can be seen that the output has a zero mean.

The design parameter can be obtained by minimizing the objective function in terms of the spectral characteristics of the covariance function of the input and output,

$\min _{a_{i}, b_{j}, \alpha_{k}, \beta_{l}}\left\|C_{E}\right\|_{2}=\min _{a_{i}, b_{j}, \alpha_{k}, \beta_{l}}\left\|C_{K_{Y Y}}-C_{K_{Y_{D} Y_{D}}}\right\|_{2}$,

where \|\|$_{2}$ denote the Euclidean norm of a matrix.

(b) Optimal controller design by parametric optimization

Assume that the system is described by Eq. (4), where coefficients $a_{i}, b_{j}$ are independent random variables with a given distribution. The set point input is a random process with a given mean and given covariance function as follows:

$M_{R}(t)=E[R(t)]=\left(C_{m_{R}}\right)^{T} \psi(t)$

$\kappa_{R R}=E\left\{\left[R\left(t_{1}\right)-M_{R}\left(t_{1}\right)\right]\left[R\left(t_{2}\right)-M_{R}\left(t_{2}\right)\right]\right\}=\sum_{i=1}^{N} \sum_{j=1}^{N} \psi_{i}\left(t_{1}\right) \psi_{j}\left(t_{2}\right) c_{i j}^{R}=\psi\left(t_{1}\right)^{T} C_{K_{R R}} \psi\left(t_{2}\right)$

The system is in the closed-loop, as shown in Fig. 1, with a fractional order $\mathrm{PI}^{\lambda} \mathrm{D}^{\mu}$ controller [18]:

$C(s)=K_{p}+\frac{K_{i}}{s^{\lambda}}+K_{d} s^{\mu}$.

The parameters of the $\mathrm{PI}^{\lambda} \mathrm{D}^{\mu}$ controller are obtained by optimizing the cost function as follows:

$$
\begin{aligned}
& \min _{K_{p}, K_{i}, K_{d}, \lambda, \mu} J=\min _{K_{p}, K_{i}, K_{d}, \lambda, \mu} E \int_{0}^{T}\left(y_{d}(t)-y(t)\right)^{2} d t \\
& =\min _{K_{p}, K_{i}, K_{d}, \lambda, \mu} \int_{0}^{T}\left\{E\left[y_{D}^{2}(t)\right]+E\left[y(t)^{2}\right]-2 E\left[y_{d}(t)\right] E[y(t)]\right\} d t .
\end{aligned}
$$

where $E\left[y(t)^{2}\right]$ and $E[y(t)]$ are obtained from Eq. (20).

Because the variance is a measure of the variability of a random process, a large variance of the system output implies a large deviation from the nominal response under stochastic input/parametric uncertainties. An additional constraint on the variance can be used in combination with the objective function (26)

$\max _{0 \leq t \leq T} D_{y}(t)=\kappa_{Y Y}(t, t) \leq D_{\max }$. 


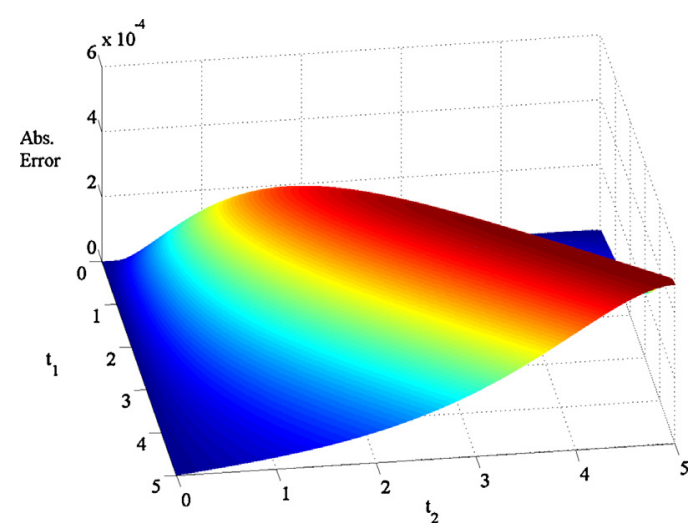

Fig. 3. Absolute error surface for the approximated covariance function with Eq. (20) and the exact desired covariance function for example 1.

The upper bound, $D_{\max }$, of the variance of the system output can be considered the maximum allowable sensitivity of the system response to the stochastic uncertainties.

\section{Examples}

\subsection{Example 1: shaping filter design}

Assume the shaping filter can be described as

$D_{0}^{\alpha} y(t)+a y(t)=b u(t)$,

where $u(t)$ is a zero mean random process with the covariance function, $\kappa_{U U}\left(t_{1}, t_{2}\right)=\delta\left(t_{1}-t_{2}\right)$, and $\delta$ is the Dirac delta function.

The desired covariance function of output can be expressed as an Ornstein-Ulenbeck process:

$\kappa_{Y_{D} Y_{D}}\left(t_{1}, t_{2}\right)=\frac{1}{2}\left(e^{-\left|t_{1}-t_{2}\right|}-e^{-\left(t_{1}+t_{2}\right)}\right) ; \quad t_{1}, t_{2} \geq 0$.

The design parameters $\alpha, a$, and $b$ are obtained by minimizing Eq. (23). The results are $\alpha=1.001 ; a=0.996 ; b=0.998$, which are sufficiently close to the exact (analytical) result, $\alpha=1 ; a=1 ; b=1$ [19].

The operational matrix for system (28) is

$A_{G}=\left(B_{\alpha}+a I\right)^{-1} b I$,

where $I$ is an identity matrix, and $B_{\alpha}$ is an operational matrix of the derivative.

1024 block pulse functions were used for this example.

Fig. 3 shows the error surface of the covariance function given by Eq. (20) for the optimal parameters with respect to the exact desired covariance function of Eq. (29). As shown in the figure, the level of relative error is small enough, $<0.5 \%$. This simple example highlights the effectiveness of the proposed method in designing the shaping filter.

\subsection{Example 2}

\subsubsection{Example $2 a$}

Consider system described by the double-fractional order differential equation,

$D_{0}^{3 / 4} y(t)+D_{0}^{1} y(t)=u(t)$

where $u(t)$ is random white noise with a zero mean covariance function, $\kappa_{U U}\left(t_{1}, t_{2}\right)=0.1 \delta\left(t_{1}-t_{2}\right)$.
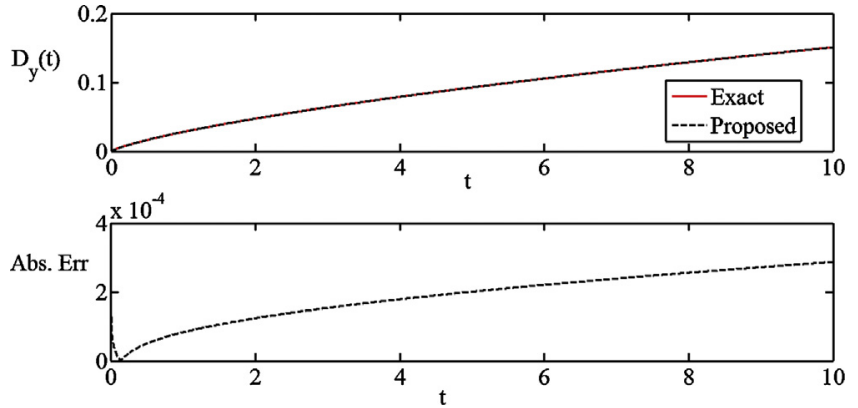

Fig. 4. Variance of the system output for example 2a and the absolute error of the proposed method.
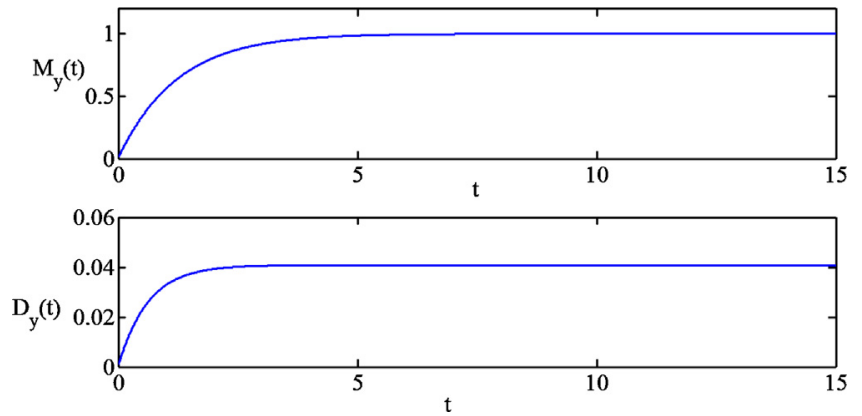

Fig. 5. Mean and variance of system output for example $2 \mathrm{~b}$ with the proposed controller.

The exact variance of the system output can be expressed as [20]

$D_{y(t)}=\sigma^{2}(t)=\frac{0.1}{a_{2}^{2}} \int_{0}^{t} u^{2\left(\alpha_{2}-1\right)}\left[\varepsilon_{\alpha_{2}-\alpha_{1}, \alpha_{2}}\left(-\frac{a_{1} u^{\left(\alpha_{2}-\alpha_{1}\right)}}{a_{2}}\right)\right]^{2} d u$,

where $\varepsilon_{\alpha_{2}-\alpha_{1}, \alpha_{2}}($ ) is a Mittag-Leffler function that can be calculated using MATLAB function mlf.m [21], $a_{1}=a_{2}=1 ; \alpha_{1}=3 / 4$, and $\alpha_{2}=1$.

1024 BPFs were used for the approximation in this example. The operational matrix for this system can be expressed as

$A_{G}=\left(B_{3 / 4}+B_{1}\right)^{-1}$.

Fig. 4 shows the variance of the system output by the proposed method and analytical variance in Eq. (32). The figure also shows the absolute error of the variance by the proposed method with respect to the analytical results. The proposed method can provide accurate results for predicting the variance of the system output.

\subsubsection{Example $2 b$}

Consider the above system in a closed loop configuration with a $\mathrm{PI}^{\lambda} \mathrm{D}^{\mu}$ controller. The set point $r(t)$ is a random process with unit mean and the same covariance function as in the previous example. A PI ${ }^{\lambda} \mathrm{D}^{\mu}$ controller is design for this system by minimizing the objective function (26) with the desired output, $y_{d}(t)=1$ (deterministic unit step response). The objective function is minimized using the pattern search function of the optimization toolbox of MATLAB.

The result parameters of controller are $K_{p}=0.8286$; $K_{i}=0.8137 ; \lambda=0.2660 ; K_{d}=0.0056 ; \mu=0.9996$. Fig. 5 shows the mean and variance of the system with the designed controller. Fig. 6 shows 100 Monte Carlo simulations of this system with the proposed controller. By comparing the variances of the system outputs in Figs. 4 and 5, the designed controller can stabilize this stochastic fractional order system. The performance of the proposed controller is satisfactory. 


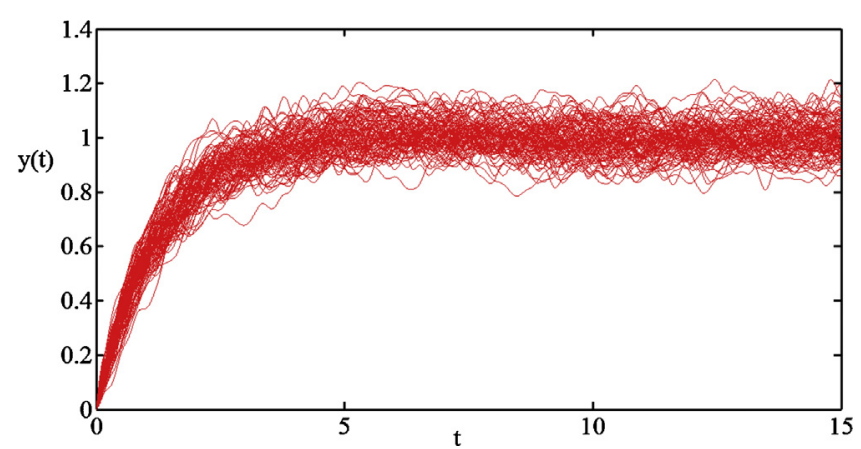

Fig. 6. $100 \mathrm{MC}$ simulation of example $2 \mathrm{~b}$.

\subsection{Example 3}

The proposed method was used to design a $\mathrm{PI}^{\lambda}$ controller for a fractional order system that has been studied in the numerical references on a fractional order system [14,22-24]:

$P(s)=\frac{k_{1}}{k_{2} s^{0.5}+1}$.

where $k_{1}$ and $k_{2}$ are uniform random variables in the interval $[0.5,1.5]$. This fractional order model in Eq. (34) is known as the Cole-Cole model, which provides a parameter of the fractional order that interprets the underlying mechanism of dielectric relaxation [22-24].

The set point input, $R(t)$, is a band-limited Gaussian white noise process with a unit mean and a covariance function of

$\kappa_{R R}\left(t_{1}, t_{2}\right)=\frac{W_{B}}{\pi} \operatorname{sinc}\left(\frac{\left(t_{1}-t_{2}\right) W_{B}}{\pi}\right)$,

with $W_{B}=0.02 \pi$, and the $\operatorname{sinc}()$ function is defined as

$\sin c(x)=\left\{\begin{array}{l}\sin (\pi x) /(\pi x) \text { elsewhere } \\ 1 \text { for } x=0\end{array}\right.$.

It can be seen from Fig. 1 that the transfer function from measurement noise $n(t)$ to output $y(t)$ is the same as the transfer function from $r(t)$ to $y(t)$, i.e., $Y(s)=T(s) R(s)-T(s) N(s)$.

Let us define a new signal $z(t)=r(t)-n(t)$. It can then be shown that:

The expectation $\bar{z}(t)=m_{z}(t)$ of $z(t)$ is equal to $r(t)$. The covariance of $z(t)$ is

$$
\begin{aligned}
& E\left\{(z-\bar{z})^{2}\right\}=E\left\{z^{2}-2 z \bar{z}+\bar{z}^{2}\right\}=E\left(z^{2}\right)-\bar{z}^{2}=E\left\{(r-n)^{2}\right\}-r^{2} . \\
& =E\left\{r^{2}+n^{2}-2 r n\right\}-r^{2}=E\left\{n^{2}\right\}
\end{aligned} .
$$

In the above equation, the dependent of signals on time is dropped; $r(t)$ and $n(t)$ are independent. The covariance of new signal is the same as the correlation function of measurement noise. Note that the covariance and correlation of the measurement noise is the same because the measurement noise is zero mean. Thus, in combination, the deterministic set point and random measurement noise can be viewed as a random set point with the mean as a deterministic input and the covariance as a measurement noise.

A PI $\lambda$ controller was obtained by minimizing the objective function (26) with the desired output is $y_{d}(t)=1$. The search space for optimal parameter of the controller was limited to $0 \leq K_{p} \leq 1 ; 0 \leq K_{i} \leq 40 ; 0.9 \leq \lambda \leq 1.5$ for the sake of simplicity, as in other studies on the probabilistic approach $[25,26]$. The initial controller, $C_{0}(s)=0.1817+\left(36.3528 / s^{1.216}\right)$, was obtained from [24]. The resulting controller parameters are

$C_{1}(s)=1+\left(40 / s^{0.9}\right)$.
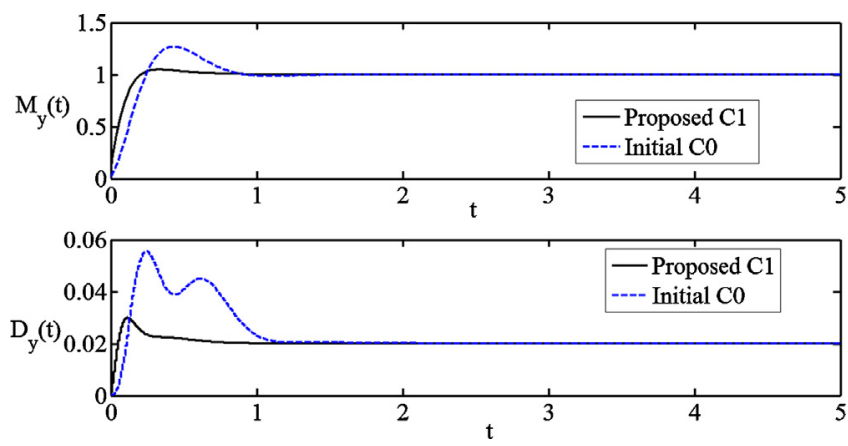

Fig. 7. Mean and variance of the system output for example 3 with the proposed and initial controllers.

Fig. 7 shows the mean and variance of the system output with the initial and proposed controllers. Fig. 8 shows a bounded region for 2000 possible responses of the uncertain system with the proposed and initial controllers. These figures show that the proposed controller outperforms the initial one under the influence of stochastic uncertainties.

\subsection{Example 4: systems with infinite $H^{2}$ norm}

\subsubsection{Example $4 a$}

Integrator is an elementary block on many control systems. It is adequate for presenting a basic inventory system in process control such as a liquid level control system $[27,28]$. Consider an integral system:

$G(s)=\frac{1}{s}$,

subject to the input with a zero mean first order Gauss Markov process with a covariance function of:

$K_{U U}\left(t_{1}, t_{2}\right)=\sigma^{2} e^{-\beta\left|t_{1}-t_{2}\right|}$.

The variance of the system output can be calculated analytically as [29]:

$\left.D_{Y Y}(t)=\frac{2 \sigma^{2}}{\beta^{2}}\left(\beta t-\sum e^{-\beta t}\right)\right)$.

The analytical result is shown in Fig. 9. The results and the absolute errors with respect to the exact variance by the hybrid spectral (proposed) and Quasi Monte Carlo (QMC) methods are also shown in Fig. 9. The errors by both the QMC and proposed methods grow with time since the output variance increases without bound as $t$ goes to infinity. The QMC method used 5000 samples of random process and the computational time needed was $117 \mathrm{~s}$ on an Intel core i3 laptop with 2GB RAM. On the other hand, the computational

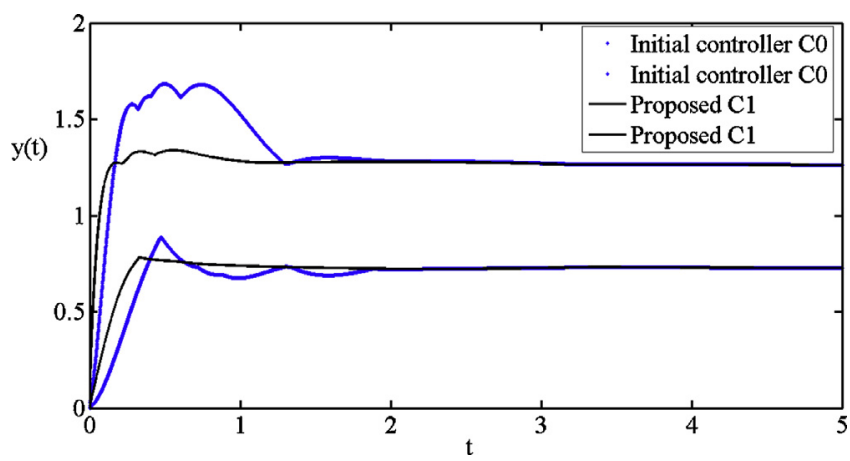

Fig. 8. Bounded regions for 2000 MC simulations of the stochastic system with the proposed and initial controllers. 

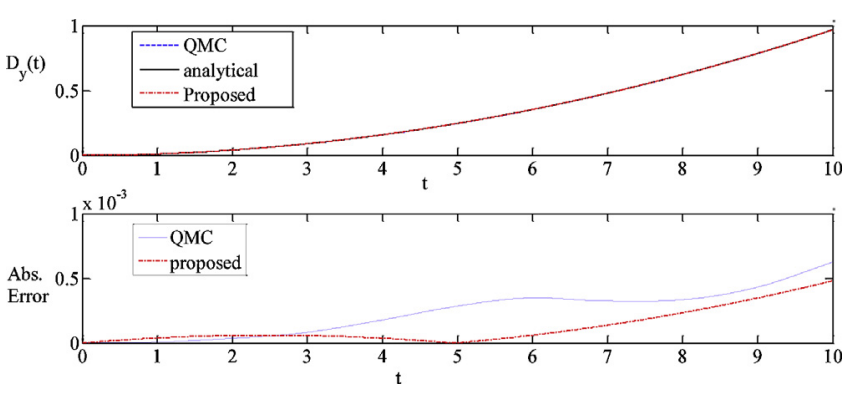

Fig. 9. Variance of system output for example 4a.

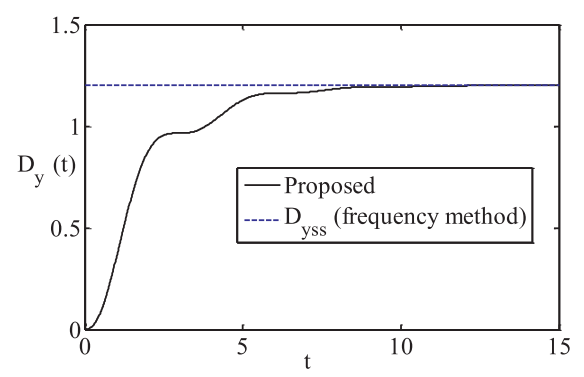

Fig. 10. Variance of system output for example $4 \mathrm{~b}$ for $K=1, v=1.7, \lambda=1$.

time of the proposed method with 2048 BPFs was only 0.73 s. Since the convergence rate of the QMC method is proportional to the magnitude of variance, the QMC method can give better accuracy at the beginning of the transient time due to the small variance at the beginning. It can be seen that the proposed method gives better performance in computational load. Hence, it is more suitable for the direct design via optimization than the QMC method. In general, the analytical output variance can be calculated only for a few particular cases, especially when a fractional order is involved.

\subsubsection{Example $4 b$}

Consider a fractional order system:

$G(s)=\frac{K}{s^{\nu}+\lambda}$,

subject to a zero mean ideal white noise with a covariance of $\delta\left(t_{1}-t_{2}\right)$. From the frequency method, the steady state variance of the output is the same as system $\mathrm{H}^{2}$ norm [30]

$D_{y s s}=\|G\|_{2}^{2}=\frac{1}{2 \pi} \int_{-\infty}^{\infty}|G(j \omega)|^{2} d \omega= \begin{cases}\infty & \text { if } 0<v \leq 1 / 2 \\ -K^{2} \lambda\left(\frac{1}{v}-2\right) \frac{\cot \left(v \frac{\pi}{2}\right)}{v \sin \left(\frac{\pi}{v}\right)} & \text { if } 1 / 2<v \leq 2 \text { and } v \neq 1 . \\ \frac{K^{2}}{2 \lambda} & \text { if } \quad v=1\end{cases}$

Fig. 10 shows the exact steady state variance of system output by the frequency method for $K=1, v=1.7, \lambda=1$. Fig. 10 also shows the variance of system output by the proposed method. It can be seen that when the $\mathrm{H}^{2}$ norm of system is finite, the proposed method gives a consistent result with the frequency method for a steady state variance.

Fig. 11 shows the variance of the output calculated by the propose method with different number of block pulse functions for $K=1, v=0.4, \lambda=1$. The frequency method gives the steady state variance $\|G\|_{2}^{2}=\infty$ by Eq. (42). It seems that the proposed method still has a limitation for obtaining the accurate result for this case. To the authors' knowledge, there is no general method available for the case where the system has a fast response and infinite $\mathrm{H}^{2}$ norm.

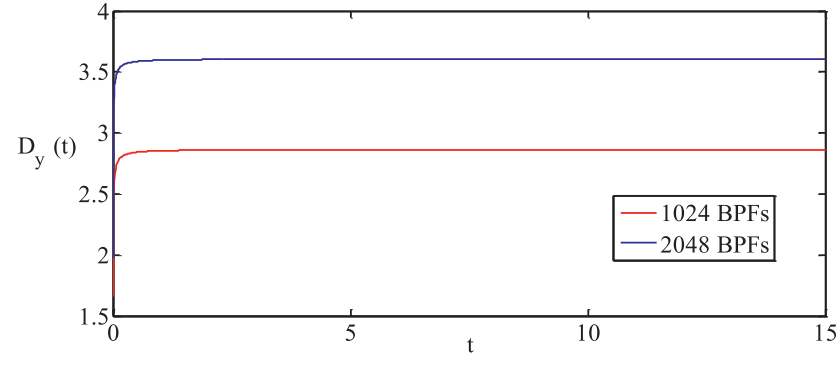

Fig. 11. Variance of system output for example $4 \mathrm{~b}$ for $K=1, v=0.4, \lambda=1$.

\section{Conclusions}

Fractional calculus has already been accepted as a powerful tool for modeling and control of complicated process [24,30-33]. In this work, an approach for the optimal design for linear fractional order processes with stochastic uncertainties was proposed. The use of the hybrid spectral method leads to algebraic relationships between the first and second order stochastic moments of a system's input and output. Therefore, the spectral method can obtain a highly accurate solution with less computational demand than the traditional Monte Carlo method. Hence, it is more suitable for the optimal design. The proposed method can be used for the synthesis colored noise from the arbitrary given noise. Moreover, the stability of uncertain systems can be inferred from a statistical evolution of the system output, and robust $\mathrm{PI}^{\lambda} \mathrm{D}^{\mu}$ controllers can be designed efficiently with respect to the stochastic uncertainties. Depending on whether the robustness or nominal performance is of primary concern, different parameter distributions and stochastic forcing can be assumed for the stochastic uncertainties. Based on the different assumptions of the stochastic uncertainties, the optimal parameters were calculated using a simple nonlinear optimization. Integer order systems can be treated as a particular case of fractional order systems. The simulation examples confirmed that the proposed design method gives robust closed-loop responses for a range of stochastic systems. Extension of the proposed to the nonlinear case is an interesting issue for future works.

\section{Acknowledgement}

This study was supported by the Basic Science Research Program through the National Research Foundation of Korea (NRF) funded by the Ministry of Education, Science and Technology (2012012532).

\section{References}

[1] W. Chen, A speculative study of 2/3-order fractional Laplacian modelling of turbulence; some though and conjectures, Chaos 16 (2006) 023126. 
[2] K. Weron, M. Kotulski, On the cole-cole relaxation functions and related Mittag-Leffler distribution, Physica A 232 (1996) 180-188.

[3] D. Baleanu, Z.B. Guvenc, T.J.A. Machado (Eds.), New Trend in Nanotechnology and Fractional Calculus Applications, Springer, Dordrecht, 2010.

[4] R.L. Magin, B.S. Akpa, T. Neuberger, A.G. Webb, Fractional order analysis of sephadex gel structure: NMR measurement reflecting anomalous diffusion, Commun. Nonlinear Sci. Numer. Simul. 16 (12)(2010) 4581-4587 (SI: Complex Systems and Chaos with Fractionality, Discontinuity Nonlinearity).

[5] M.K. Bouafoura, N.B. Braiek, $\mathrm{PI}^{\lambda} \mathrm{D}^{\mu}$ controller design for integer and fractional plants using piecewise orthogonal functions, Commun. Nonlinear Sci. Numer. Simul. 15 (5) (2010) 1267-1278.

[6] B.M. Vinagre, V. Feliu, J.J. Feliu, Frequency domain identification of a flexible structure with piezoelectric actuator using irrational transfer functions, in: Proc. 37th IEEE Conf. Decision and Control, Tampa, FL, 1998.

[7] R. Kalges, G. Radons, M. Sokolov (Eds.), Anomalous Transport: Foundations and Applications, Wiley VCH, Weinheim, 2008

[8] F. Mainardi, Fractional Calculus and Waves in Linear Viscoelasticity, Imperial College Press, London, 2010.

[9] L. Dunn, J.K. Shultis, Exploring Monte Carlo Methods, Elsevier, San Diego, 2011.

[10] K.A. Pupkov, N.D. Egupov (Eds.), Classical and Modern Theory of Control Systems V2, BMSTU Press, Moscow, 2003.

[11] D. Xiu, Numerical Method for Stochastic Computation: Spectral Approach, Princeton University Press, New Jersey, 2011.

[12] D. Xiu, G. Karniadakis, The Wiener-Askey polynomial chaos for stochastic differential equation, SIAM J. Sci. Comput. 24 (2) (2003) 619-644.

[13] K.A. Pupkov, N.D. Egupov, A.M. Makarenkov, A.I. Trofimov, Theory and Numer ical Methods for Studying Stochastic Systems, Fizmatlits, Moscow, 2003.

[14] P.L.T. Duong, M. Lee, Uncertainty propagation in stochastic fractional order processes using spectral methods: a hybrid approach, Commun. Nonlinear Sci. Numer. Simul. 17 (11) (2012) 4262-4273.

[15] A.C. Monje, Y. Chen, M.B. Vingare, D. Xue, V. Feliu, Fractional-order Systems and Controls, Springer, London, 2010

[16] C.H. Wang, On the generalization of block pulse operational matrix for fractional calculus, J. Franklin Inst. 315 (2) (1983) 91-102.

[17] A. Lofti, S.A. Yousefi, M. Dehghan, Numerical solution of a class of fractiona optimal control problems via Legendre orthonormal basis combined with the operational matrix and the Gauss quadrature, J. Comput. Appl. Math. 250 (2013) 143-160.
[18] I. Podlubny, Fractional-order system and $\mathrm{PI}^{\lambda} \mathrm{D}^{\mu}$ controllers, IEEE Trans. Autom. Control 44 (1) (1999) 208-214

[19] L. Garcia, Probability, Statistics and Random Processes for Electrical Engineering, 3rd Pearson Education, Inc., Upper Saddle River, NJ07458, 2008.

[20] C.H. Eab, S.C. Lim, Fractional Langevin equations of distributed order, Phys. Rev. E 83 (2011) 031136.

[21] I. Podlubny, M. Kacenak, http://www.mathworks.com/matlabcentral/ fileexchange/8738-mittag-leffler-function

[22] I. Gochyuk, P. Hangi, Fractional diffusion modeling of ion channel gating, Phys Rev. E 70 (2004) 051915.

[23] L. Margin, Fractional Calculus in Bioengineering, Begell House Publisher Inc. Redding, 2006

[24] Y. Luo, Y. Chen, C. Wang, G. Pi, Tuning fractional order proportional integra controllers, J. Process Control 20 (2010) 823-883.

[25] R. Tempo, G. Caliafore, F. Dabbene, Randomized Algorithm for Analysis and Control of Uncertain Systems, 2nd ed., Springer-Verlag, London, 2013.

[26] P.L.T. Duong, M. Lee, Robust PID controller design for processes with stochastic parametric uncertainties, J. Process Control 22 (2012) 1559-1566.

[27] V.H. Nguyen, Y. Yamashita, M. Lee, Optimization based approach for industrial PI controller design for optimal servo control of integrating process with constraints, J. Chem. Eng. Jpn. 44 (2011) 345-354.

[28] D.E. Seborg, T.F. Edgar, Process Dynamic and Control, 2nd ed., John Wiley and Sons, New York, 2004.

[29] R.G. Brown, P.Y.C. Hwang, Introduction Random Signals and Applied Kalman Filtering: With MATLAB Exercises, 4th ed., John Wiley \& Sons, Hoboken, 2012.

[30] R. Malti, M. Aoun, F. Levron, A. Oustaloop, Analytical computation of $\mathrm{H}^{2}$ norm of fractional commensurate transfer function, Automatica 47 (2011) 2425-2432.

[31] M. Tavakoli-Kakhi, M.S. Tavazoei, Proportional stabilization and closed loop identification of an unstable fractional order process, J. Process control 24 (2014) 542-549.

[32] V. Feliu-Batlle, R.R. Perez, F.J. Castillo Garcia, L. Sanchez Rodriguez, Smith predictor based robust fractional order control: application to water distribution in a main irrigation canal pool, J. Process control 19 (2009) 506-519.

[33] F.J. Castillo Garcia, V. Feliu-Batlle, R.R. Perez, Frequency specification regions of fractional-order PI controller for first order plus time delay processes, J. Process control 23 (2013) 598-612. 